Volume 70, Number 2, Pages 702-718(2021)

DOI: $10.31801 /$ cfsuasmas. 866753

ISSN 1303-5991 E-ISSN 2618-6470

Received by the editors: January 22, 2021; Accepted: April 3, 2021

\title{
ON THE RESOLVENT OF SINGULAR $q$-STURM-LIOUVILLE OPERATORS
}

\author{
Bilender P. ALLAHVERDİEV ${ }^{1}$ and Hüseyin TUNA ${ }^{2}$ \\ ${ }^{1}$ Department of Mathematics, Süleyman Demirel University, \\ 32260 Isparta, TURKEY \\ ${ }^{2}$ Department of Mathematics, Mehmet Akif Ersoy University, \\ 15030 Burdur, TURKEY
}

\begin{abstract}
In this paper, we investigate the resolvent operator of the singular $q$ Sturm-Liouville problem defined as

$$
-\frac{1}{q} D_{q^{-1}}\left[D_{q} y(x)\right]+[r(x)-\lambda] y(x)=0,
$$

with the boundary condition

$$
y(0, \lambda) \cos \beta+D_{q^{-1}} y(0, \lambda) \sin \beta=0,
$$

where $\lambda \in \mathbb{C}, r$ is a real-valued function defined on $[0, \infty)$, continuous at zero and $r \in L_{q, l o c}^{1}[0, \infty)$. We give a representation for the resolvent operator and investigate some properties of this operator. Furthermore, we obtain a formula for the Titchmarsh-Weyl function of the singular $q$-Sturm-Liouville problem.
\end{abstract}

\section{INTRODUCTION}

Quantum (or $q$ ) calculus is a very interesting field in mathematics. It has numerous in statistic physics, quantum theory, the calculus of variations and number theory; see, e.g., 12, 1, 11, 14, 15, 18, 21, 24]). The first results in $q$-calculus belong to the Euler. In 2005, Annaby and Mansour investigated $q$-Sturm-Liouville problems [10]. Later in [9], the authors studied the Titchmarsh-Weyl theory for $q$-Sturm-Liouville equations. In [3 4], the authors proved the existence of a spectral function for $q$-Sturm-Liouville operator.

In this article, we investigate the following $q$-Sturm-Liouville problem defined as

$$
-\frac{1}{q} D_{q^{-1}} D_{q} y(x)+u(x) y(x)=\lambda y(x),
$$

2020 Mathematics Subject Classification. 33D15, 34L40, 39A13, 34L10.

Keywords and phrases. q-Sturm-Liouville operator, spectral function, resolvent operator, Titchmarsh-Weyl function.

\bilenderpasaoglu@sdu.edu.tr; hustuna@gmail.com-Corresponding author

(1D)0000-0002-9315-4652; 0000-0001-7240-8687. 
where $0<x<\infty$. The resolvent operator for this problem is constructed. Using the spectral function, an integral representation is obtained. Furthermore, some properties of this operator are investigated. A formula for the Titchmarsh-Weyl function of Eq. (1) is given. Historically, in 1910, H. Weyl was first obtained a representation theorem for the resolvent of Sturm-Liouville problem defined by

$$
-\left(p y^{\prime}\right)^{\prime}+q y=\lambda y, x \in(0, \infty),
$$

where $p, q$ are real-valued and $p^{-1}, q \in L_{l o c}^{1}[0, \infty)$. Similar representation theorems were proved in $25,20,2,5,6,7.7$.

\section{Preliminaries}

In this section, we give a brief introduction to quantum calculus and refer the interested reader to $17,8,12$.

Let $0<q<1$ and let $A \subset \mathbb{R}$ is a $q$-geometric set, i.e., $q x \in A$ for all $x \in A$. The Jackson q-derivative is defined by

$$
D_{q} y(x)=\mu^{-1}(x)[y(q x)-y(x)],
$$

where $\mu(x)=q x-x$ and $x \in A$. We note that there is a connection the Jackson $q$ derivative between and $q$-deformed Heisenberg uncertainty relation (see 23$]$ ). The $q$-derivative at zero is defined as

$$
D_{q} y(0)=\lim _{n \rightarrow \infty}\left[q^{n} x\right]^{-1}\left[y\left(q^{n} x\right)-y(0)\right] \quad(x \in A),
$$

if the limit in 22) exists and does not depend on $x$. The Jackson q-integration is given by

$$
\int_{0}^{x} f(t) d_{q} t=x(1-q) \sum_{n=0}^{\infty} q^{n} f\left(q^{n} x\right) \quad(x \in A),
$$

provided that the series converges, and

$$
\int_{a}^{b} f(t) d_{q} t=\int_{0}^{b} f(t) d_{q} t-\int_{0}^{a} f(t) d_{q} t,
$$

where $a, b \in A$. The $q$-integration for a function over $[0, \infty)$ defined by the formula ( [13])

$$
\int_{0}^{\infty} f(t) d_{q} t=\sum_{n=-\infty}^{\infty} q^{n} f\left(q^{n}\right) .
$$

Let $f$ be a function on $A$ and let $0 \in A$. For every $x \in A$, if

$$
\lim _{n \rightarrow \infty} f\left(x q^{n}\right)=f(0),
$$

then $f$ is called $q$-regular at zero. Throughout the paper, we deal only with functions $q$-regular at zero. 
The following relation holds

$$
\int_{0}^{a} g(t) D_{q} f(t) d_{q} t+\int_{0}^{a} f(q t) D_{q} g(t) d_{q} t=f(a) g(a)-f(0) g(0),
$$

where $f$ and $g$ are $q$-regular at zero.

Let $L_{q}^{2}[0, \infty)$ be the Hilbert space consisting of all functions $f$ satisfying $([9])$

$$
\|f\|:=\sqrt{\int_{0}^{\infty}|f(x)|^{2} d_{q} x}<+\infty
$$

with the inner product

$$
(f, g):=\int_{0}^{\infty} f(x) \overline{g(x)} d_{q} x .
$$

The $q$-Wronskian of the functions $y($.$) and z($.$) is defined by the formula$

$$
W_{q}(y, z)(x):=y(x) D_{q} z(x)-z(x) D_{q} y(x),
$$

where $x \in[0, \infty)$.

\section{Main Results}

Consider the $q$-Sturm-Liouville equation

$$
L(y):=-\frac{1}{q} D_{q^{-1}} D_{q} y(x)+r(x) y(x)=\lambda y(x),
$$

satisfying the conditions

$$
\begin{gathered}
y(0, \lambda) \cos \beta+D_{q^{-1}} y(0, \lambda) \sin \beta=0, \\
y\left(q^{-n}, \lambda\right) \cos \alpha+D_{q^{-1}} y\left(q^{-n}, \lambda\right) \sin \alpha=0, \alpha, \beta \in \mathbb{R}, n \in \mathbb{N}:=\{1,2, \ldots\},
\end{gathered}
$$

where $\lambda \in \mathbb{C}, r$ is a real-valued function defined on $[0, \infty)$, continuous at zero and $r \in L_{q, l o c}^{1}[0, \infty)$.

Let $\varphi(x, \lambda)$ and $\theta(x, \lambda)$ be the solutions of the Eq. (3) satisfying the following conditions

$$
\begin{aligned}
& \varphi(0, \lambda)=\sin \beta, D_{q^{-1}} \varphi(0, \lambda)=-\cos \beta, \\
& \theta(0, \lambda)=\cos \beta, D_{q^{-1}} \theta(0, \lambda)=\sin \beta .
\end{aligned}
$$

Lemma 1 ( 9$]$ ). Let $\lambda \notin \mathbb{R}$ and let

$$
\chi_{q^{-n}}(x, \lambda)=\theta(x, \lambda)+l\left(\lambda, q^{-n}\right) \varphi(x, \lambda) \in L_{q}^{2}(0, \infty),
$$

where $n \in \mathbb{N}$. Then we have

$$
\begin{aligned}
\chi_{q^{-n}}(x, \lambda) & \rightarrow \chi(x, \lambda) \\
\int_{0}^{q^{-n}}\left|\chi_{q^{-n}}(q t, \lambda)\right|^{2} d_{q} x & \rightarrow \int_{0}^{\infty}|\chi(x, \lambda)|^{2} d_{q} x, n \rightarrow \infty .
\end{aligned}
$$


Putting

$$
\begin{gathered}
G_{q^{-n}}(x, t, \lambda)= \begin{cases}\chi_{q^{-n}}(x, \lambda) \varphi(t, \lambda), & t \leq x \\
\varphi(x, \lambda) \chi_{q^{-n}}(t, \lambda), & t>x,\end{cases} \\
y(x, \lambda):=\left(R_{q^{-n}} f\right)(x, \lambda)= \\
\int_{0}^{q^{-n}} G_{q^{-n}}(x, t, \lambda) f(t) d_{q} t,(\lambda \in \mathbb{C}, \operatorname{Im} \lambda \neq 0),
\end{gathered}
$$

where $f \in L_{q}^{2}\left[0, q^{-n}\right]$. Now, we shall show that the equality 77 satisfies the equation $L(y)-\lambda y(x)=f(x), x \in\left(0, q^{-n}\right)(\lambda \in \mathbb{C}, \operatorname{Im} \lambda \neq 0)$ and the boundary conditions (4)-(5). From (7), we get

$$
\begin{aligned}
y(x, \lambda)= & q \chi_{q^{-n}}(x, \lambda) \int_{0}^{x} \varphi(q t, \lambda) f(q t) d_{q} t \\
& +q \varphi(x, \lambda) \int_{x}^{q^{-n}} \chi_{q^{-n}}(q t, \lambda) f(q t) d_{q} t .
\end{aligned}
$$

From (8), it follows that

$$
\begin{aligned}
D_{q} y(x, \lambda)= & q D_{q} \chi_{q^{-n}}(x, \lambda) \int_{0}^{x} \varphi(q t, \lambda) f(q t) d_{q} t \\
& +q D_{q} \varphi(x, \lambda) \int_{x}^{q^{-n}} \chi_{q^{-n}}(q t, \lambda) f(q t) d_{q} t,
\end{aligned}
$$

and

$$
\begin{aligned}
D_{q^{-1}} D_{q} y(x, \lambda)= & q D_{q^{-1}} D_{q} \chi_{q^{-n}}(x, \lambda) \int_{0}^{x} \varphi(q t, \lambda) f(q t) d_{q} t \\
& +q D_{q^{-1}} D_{q} \varphi(x, \lambda) \int_{x}^{q^{-n}} \chi_{q^{-n}}(q t, \lambda) f(q t) d_{q} t \\
& -q W_{q}\left(\chi_{q^{-n}}, \varphi\right) f(x) .
\end{aligned}
$$

Hence, by $W_{q}\left(\varphi, \chi_{q^{-n}}\right)=1(n \in \mathbb{N})$, we deduce that

$$
\begin{aligned}
& -\frac{1}{q} D_{q^{-1}} D_{q} y(x, \lambda) \\
= & (\lambda-r(x)) q \chi_{q^{-n}}(x, \lambda) \int_{0}^{x} \varphi(q t, \lambda) f(q t) d_{q} t \\
& +(\lambda-r(x)) q \varphi(x, \lambda) \int_{x}^{q^{-n}} \chi_{q^{-n}}(q t, \lambda) f(q t) d_{q} t+f(x) \\
= & (\lambda-r(x)) y(x, \lambda)+f(x),
\end{aligned}
$$


i.e., the function $y(x, \lambda)$ satisfies the equation $L(y)-\lambda y(x)=f(x), x \in\left(0, q^{-n}\right)$.

Moreover,

$$
\begin{aligned}
y(0, \lambda) & =q \varphi(0, \lambda) \int_{0}^{q^{-n}} \chi_{q^{-n}}(q t, \lambda) f(q t) d_{q} t \\
& =q \cos \beta \int_{0}^{q^{-n}} \chi_{q^{-n}}(q t, \lambda) f(q t) d_{q} t, \\
D_{q^{-1}} y(0, \lambda) & =q D_{q^{-1}} \varphi(0, \lambda) \int_{0}^{q^{-n}} \chi_{q^{-n}}(q t, \lambda) f(q t) d_{q} t \\
& =-q \sin \beta \int_{0}^{q^{-n}} \chi_{q^{-n}}(q t, \lambda) f(q t) d_{q} t,
\end{aligned}
$$

i.e., $y(x, \lambda)$ satisfies (4). Similarly, we may infer that $y(x, \lambda)$ satisfies (5).

Note that the problem (3)-(5) has a purely discrete spectrum [10].

Let $\lambda_{m, q^{-n}}$ be the eigenvalues of the problem (3)-(5). Let $\varphi_{m, q^{-n}}$ be the corresponding eigenfunctions and

$$
\alpha_{m, q^{-n}}:=\left\|\varphi_{m, q^{-n}}\right\|=\left(\int_{0}^{q^{-n}} \varphi_{m, q^{-n}}^{2}(x) d_{q} x\right)^{\frac{1}{2}}
$$

where $\varphi_{m, q^{-n}}(x):=\varphi_{m, q^{-n}}\left(x, \lambda_{m, q^{-n}}\right)$ and $m \in \mathbb{N}$.

Then we have the following Parseval equality (see [8])

$$
\int_{0}^{q^{-n}}|f(x)|^{2} d_{q} x=\sum_{m=1}^{\infty} \frac{1}{\alpha_{m, q^{-n}}^{2}}\left\{\int_{0}^{q^{-n}} f(x) \varphi_{m, q^{-n}}(x) d_{q} x\right\}^{2},
$$

where $f(.) \in L_{q}^{2}\left[0, q^{-n}\right]$.

Now, let us define the nondecreasing step function $\varrho_{q^{-n}}$ on $[0, \infty)$ by

$$
\varrho_{q^{-n}}(\lambda)=\left\{\begin{array}{cc}
-\sum_{\lambda<\lambda_{m, q^{-n}}<0} \frac{1}{\alpha_{m, q^{-n}}^{2}}, & \text { for } \lambda \leq 0 \\
\sum_{0 \leq \lambda_{m, q^{-n}}<\lambda} \frac{1}{\alpha_{m, q^{-n}}^{2}} & \text { for } \lambda>0 .
\end{array}\right.
$$

It follows from (9) that

$$
\int_{0}^{q^{-n}}|f(x)|^{2} d_{q} x=\int_{-\infty}^{\infty} F^{2}(\lambda) d \varrho_{q^{-n}}(\lambda),
$$

where

$$
F(\lambda)=\int_{0}^{q^{-n}} f(x) \varphi(x, \lambda) d_{q} x
$$


Lemma 2. Let $\kappa>0$. Then the following relation holds

$$
\stackrel{\kappa}{V}_{-\kappa}^{\kappa}\left\{\varrho_{q^{-n}}(\lambda)\right\}=\sum_{-\kappa \leq \lambda_{m, q^{-n}}<\kappa} \frac{1}{\alpha_{m, q^{-n}}^{2}}=\varrho_{q^{-n}}(\kappa)-\varrho_{q^{-n}}(-\kappa)<\Upsilon,
$$

where $\Upsilon=\Upsilon(\kappa)$ is a positive constant not depending on $q^{-n}$.

Proof. Let $\sin \beta \neq 0$. Since $\varphi(x, \lambda)$ is continuous at zero, by condition $\varphi(0, \lambda)=$ $\sin \beta$, there exists a positive number $h$ and nearby 0 such that

$$
|\varphi(x, \lambda)|>\frac{1}{\sqrt{2}}|\sin \beta|, 0 \leq x \leq h
$$

and

$$
\left(\frac{1}{h} \int_{0}^{h} \varphi(x, \lambda) d_{q} x\right)^{2}>\left(\frac{1}{\sqrt{2} h} \sin \beta \int_{0}^{h} d_{q} x\right)^{2}=\frac{1}{2} \sin ^{2} \beta .
$$

Let us define $f_{h}(x)$ by

$$
f_{h}(x)=\left\{\begin{array}{lc}
0, & x>h \\
\frac{1}{h}, & 0 \leq x \leq h .
\end{array}\right.
$$

It follows from 100 and 12 that

$$
\begin{aligned}
\int_{0}^{h} f_{h}^{2}(x) d_{q} x & =\frac{1}{h}=\int_{-\infty}^{\infty}\left(\frac{1}{h} \int_{0}^{h} \varphi(x, \lambda) d_{q} x\right)^{2} d \varrho_{q^{-n}}(\lambda) \\
& \geq \int_{-\kappa}^{\kappa}\left(\frac{1}{h} \int_{0}^{h} \varphi(x, \lambda) d_{q} x\right)^{2} d \varrho_{q^{-n}}(\lambda) \\
& >\frac{1}{2} \sin ^{2} \beta\left\{\varrho_{q^{-n}}(\kappa)-\varrho_{q^{-n}}(-\kappa)\right\},
\end{aligned}
$$

which proves the inequality (11).

Let $\sin \beta=0$ and

$$
f_{h}(x)=\left\{\begin{array}{cc}
0, & x>h \\
\frac{1}{h^{2}}, & 0 \leq x \leq h .
\end{array}\right.
$$

By 10, we can get the desired result.

We now return to the formula (7), whose right-hand side has been called the resolvent. The resolvent is known to exist for all $\lambda$ which are not eigenvalues of the problem (3)-(5). Now, we will get the expansion of the resolvent.

Since the function $y(x, \lambda)$ satisfies the equation $L(y)-\lambda y(x)=f(x), x \in\left(0, q^{-n}\right)$ $\left(\lambda \in \mathbb{C}, \lambda \neq \lambda_{m, q^{-n}}, m \in \mathbb{N}\right.$ ) and the boundary conditions (4), (5), via the $q$ integration by parts, we find (the operator $A$ generated by the expression $L$ and the boundary conditions (4), (5) is a self-adjoint (see [10]))

$$
\left(A y, \varphi_{m, q^{-n}}\right)
$$




$$
\begin{aligned}
& =\int_{0}^{q^{-n}}\left[-\frac{1}{q} D_{q^{-1}} D_{q} y(x, \lambda)+r(x) y(x, \lambda)\right] \varphi_{m, q^{-n}}(x) d_{q} x \\
& =\left(y, A \varphi_{m, q^{-n}}\right) \\
& =\int_{0}^{q^{-n}} y(x, \lambda)\left[-\frac{1}{q} D_{q^{-1}} D_{q} \varphi_{m, q^{-n}}(x)+r(x) \varphi_{m, q^{-n}}(x)\right] d_{q} x \\
& =\lambda_{m, q^{-n}} \int_{0}^{q^{-n}} y(x, \lambda) \varphi_{m, q^{-n}}(x) d_{q} x .
\end{aligned}
$$

The set of all eigenfunctions $\frac{\varphi_{m, q^{-n}}(x)}{\alpha_{m, q^{-n}}}(m \in \mathbb{N})$ of the self-adjoint operator $A$ form an orthonormal basis for $L_{q}^{2}\left(0, q^{-n}\right)$ (see 10]). Then, the function $y(., \lambda) \in$ $L_{q}^{2}\left(0, q^{-n}\right)\left(\lambda \in \mathbb{C}, \lambda \neq \lambda_{m, q^{-n}}, m \in \mathbb{N}\right)$ can be expanded into Fourier series of eigenfunctions $\frac{\varphi_{m, q^{-n}}(x)}{\alpha_{m, q^{-n}}}(m \in \mathbb{N})$ of the problem $\sqrt{3}-\sqrt{5}$ (or of the operator $A$ ). Then we have

$$
y(x, \lambda)=\sum_{m=1}^{\infty} t_{m}(\lambda) \frac{\varphi_{m, q^{-n}}(x)}{\alpha_{m, q^{-n}}},
$$

where $t_{m}(\lambda)$ is the Fourier coefficient, i.e.,

$$
t_{m}(\lambda)=\int_{0}^{q^{-n}} y(x, \lambda) \frac{\varphi_{m, q^{-n}}(x)}{\alpha_{m, q^{-n}}} d_{q} x, m \in \mathbb{N} .
$$

Since $y(x, \lambda)\left(\lambda \in \mathbb{C}, \lambda \neq \lambda_{m, q^{-n}}, m \in \mathbb{N}\right)$ satisfies the equation

$$
-\frac{1}{q} D_{q^{-1}} D_{q} y(x, \lambda)+(r(x)-\lambda) y(x, \lambda)=f(x), x \in\left(0, q^{-n}\right),
$$

we get

$$
\begin{aligned}
a_{m} & :=\int_{0}^{q^{-n}} f(x) \frac{\varphi_{m, q^{-n}}(x)}{\alpha_{m, q^{-n}}} d_{q} x \\
& =\int_{0}^{q^{-n}}\left[-\frac{1}{q} D_{q^{-1}} D_{q} y(x, \lambda)+(r(x)-\lambda) y(x, \lambda)\right] \frac{\varphi_{m, q^{-n}}(x)}{\alpha_{m, q^{-n}}} d_{q} x \\
& =\int_{0}^{q^{-n}}\left[-\frac{1}{q} D_{q^{-1}} D_{q} \varphi_{m, q^{-n}}(x)+(r(x)-\lambda) \varphi_{m, q^{-n}}(x)\right] \frac{y(x, \lambda)}{\alpha_{m, q^{-n}}} d_{q} x \\
& =\int_{0}^{q^{-n}}\left[\lambda_{m, q^{-n}} \varphi_{m, q^{-n}}(x)-\lambda \varphi_{m, q^{-n}}(x)\right] \frac{y(x, \lambda)}{\alpha_{m, q^{-n}}} d_{q} x \\
& =\lambda_{m, q^{-n}} t_{m}(\lambda)-\lambda t_{m}(\lambda), m \in \mathbb{N} .
\end{aligned}
$$


Thus, we have

and

$$
t_{m}(\lambda)=\frac{a_{m}}{\lambda_{m, q^{-n}}-\lambda}
$$

$$
\begin{aligned}
y(x, \lambda) & =\int_{0}^{q^{-n}} G_{q^{-n}}(x, t, \lambda) f(t) d_{q} t \\
& =\sum_{m=1}^{\infty} \frac{a_{m}}{\lambda_{m, q^{-n}}-\lambda} \frac{\varphi_{m, q^{-n}}(x)}{\alpha_{m, q^{-n}}}\left(\lambda \in \mathbb{C}, \lambda \neq \lambda_{m, q^{-n}}, m \in \mathbb{N}\right) .
\end{aligned}
$$

Then

$$
\begin{aligned}
& y(x, z)=\left(R_{q^{-n}} f\right)(x, z) \\
= & \sum_{m=1}^{\infty} \frac{\varphi_{m, q^{-n}}(x)}{\alpha_{m, q^{-n}}^{2}\left(\lambda_{m, q^{-n}}-z\right)} \int_{0}^{q^{-n}} f(t) \varphi_{m, q^{-n}}(t) d_{q} t \\
= & \int_{-\infty}^{\infty} \frac{\varphi(x, \lambda)}{\lambda-z}\left\{\int_{0}^{q^{-n}} f(t) \varphi_{m, q^{-n}}(t, \lambda) d_{q} t\right\} d \varrho_{q^{-n}}(\lambda) .
\end{aligned}
$$

Lemma 3. The following formula holds

$$
\int_{-\infty}^{\infty}\left|\frac{\varphi(x, \lambda)}{\lambda-z}\right|^{2} d \varrho_{q^{-n}}(\lambda)<K
$$

where $x$ is a fixed number and $z$ is a non-real number.

Proof. Let $f(t)=\frac{\varphi_{m, q^{-n}}(t)}{\alpha_{m, q^{-n}}}$. By 13 , we conclude that

$$
\frac{1}{\alpha_{m, q^{-n}}} \int_{0}^{q^{-n}} G_{q^{-n}}(x, t, z) \varphi_{m, q^{-n}}(t) d_{q} t=\frac{\varphi_{m, q^{-n}}(x)}{\alpha_{m, q^{-n}}\left(\lambda_{m, q^{-n}}-z\right)} .
$$

Under (15) and (9), we see that

$$
\begin{aligned}
\int_{0}^{q^{-n}}\left|G_{q^{-n}}(x, t, z)\right|^{2} d_{q} t & =\sum_{m=1}^{\infty} \frac{\left|\varphi_{m, q^{-n}}(x)\right|^{2}}{\alpha_{m, q^{-n}}^{2}\left|\lambda_{m, q^{-n}}-z\right|^{2}} \\
& =\int_{-\infty}^{\infty}\left|\frac{\varphi(x, \lambda)}{\lambda-z}\right|^{2} d \varrho_{q^{-n}}(\lambda) .
\end{aligned}
$$

It follows from Lemma 1 that the last integral is convergent. The proof is complete

Now, we present below for the convenience of the reader. 
Theorem $4([19])$. Let $\left(w_{n}\right)_{n \in \mathbb{N}}$ be a uniformly bounded sequence of real nondecreasing function on a finite interval $[a, b]$. Then

(i) there exists a subsequence $\left(w_{n_{k}}\right)_{k \in \mathbb{N}}$ and a non-decreasing function $w$ such that

$$
\lim _{k \rightarrow \infty} w_{n_{k}}(\lambda)=w(\lambda),
$$

where $a \leq \lambda \leq b$.

(ii) suppose

$$
\lim _{n \rightarrow \infty} w_{n}(\lambda)=w(\lambda),
$$

where $a \leq \lambda \leq b$. Then, we have

$$
\lim _{n \rightarrow \infty} \int_{a}^{b} f(\lambda) d w_{n}(\lambda)=\int_{a}^{b} f(\lambda) d w(\lambda),
$$

where $f \in C[a, b]$.

By Lemma 2 and Theorem 4, one can find a sequence $\left\{q^{-n_{k}}\right\}$ such that

$$
\lim _{k \rightarrow \infty} \varrho_{q^{-n_{k}}}(\lambda) \rightarrow \varrho(\lambda),
$$

where $\varrho(\lambda)$ is a monotone function.

Lemma 5. Let $z \notin \mathbb{R}$. Then we have

$$
\int_{-\infty}^{\infty}\left|\frac{\varphi(x, \lambda)}{\lambda-z}\right|^{2} d \varrho(\lambda) \leq K,
$$

where $x$ is a fixed number.

Proof. Let $\eta>0$. It follows from (14) that

$$
\int_{-\eta}^{\eta}\left|\frac{\varphi(x, \lambda)}{\lambda-z}\right|^{2} d \varrho_{q^{-n}}(\lambda)<K .
$$

Then

$$
\int_{-\infty}^{\infty}\left|\frac{\varphi(x, \lambda)}{\lambda-z}\right|^{2} d \varrho(\lambda)=\lim _{\substack{\eta \rightarrow \infty \\ n \rightarrow \infty}} \int_{-\eta}^{\eta}\left|\frac{\varphi(x, \lambda)}{\lambda-z}\right|^{2} d \varrho_{q^{-n}}(\lambda)<K .
$$

Lemma 6. Let $\eta>0$. Then we have

$$
\int_{-\infty}^{-\eta} \frac{d \varrho(\lambda)}{|\lambda-z|^{2}}<\infty, \int_{\eta}^{\infty} \frac{d \varrho(\lambda)}{|\lambda-z|^{2}}<\infty .
$$

Proof. Let $\sin \beta \neq 0$. From (16), we deduce that

$$
\int_{-\infty}^{\infty} \frac{d \varrho(\lambda)}{|\lambda-z|^{2}}<\infty
$$


Let $\sin \beta=0$. Hence we see that

$$
\frac{1}{\alpha_{m, q^{-n}}} \int_{0}^{q^{-n}} \varphi_{m, q^{-n}}(t) D_{q, x}\left[G_{q^{-n}}(x, t, z)\right] d_{q} t=\frac{D_{q, x} \varphi_{m, q^{-n}}(x)}{\alpha_{m, q^{-n}}\left(\lambda_{m, q^{-n}}-z\right)} .
$$

It follows from (9) that

$$
\int_{0}^{q^{-n}}\left|D_{q, x}\left[G_{q^{-n}}(x, t, z)\right]\right|^{2} d_{q} t=\int_{-\infty}^{\infty}\left|\frac{D_{q, x} \varphi(x, \lambda)}{\lambda-z}\right|^{2} d \varrho_{q^{-n}}(\lambda) .
$$

Proceeding similarly, we can get the desired result.

Lemma 7. Let

$$
G(x, t, z)= \begin{cases}\chi(x, z) \varphi(t, z), & x \geq t \\ \varphi(x, z) \chi(t, z), & x<t\end{cases}
$$

and let $f(.) \in L_{q}^{2}[0, \infty)$. Then we have

$$
\int_{0}^{\infty}|(R f)(x, z)|^{2} d_{q} x \leq \frac{1}{v^{2}} \int_{0}^{\infty}|f(x)|^{2} d_{q} x
$$

where

$$
(R f)(x, z)=\int_{0}^{\infty} G(x, t, z) f(t) d_{q} t,
$$

and $z=u+i v$.

Proof. See 9].

Now we shall state the main result of this paper.

Theorem 8. The following relation holds

$$
(R f)(x, z)=\int_{-\infty}^{\infty} \frac{\varphi(x, \lambda)}{\lambda-z} F(\lambda) d \varrho(\lambda),
$$

where $f(.) \in L_{q}^{2}[0, \infty)$,

$$
F(\lambda)=\lim _{\xi \rightarrow \infty} \int_{0}^{q^{-\xi}} f(x) \varphi(x, \lambda) d_{q} x,
$$

and $z \notin \mathbb{R}$.

Proof. Define the function $f_{\xi}(x)$ as

$$
f_{\xi}(x)=\left\{\begin{array}{cc}
f_{\xi}(x), & x \in\left[0, q^{-\xi}\right], \\
0, & x \notin\left[0, q^{-\xi}\right]
\end{array} \quad\left(q^{-\xi}<q^{-n}\right)\right.
$$

such that $f_{\xi}(x)$ satisfies (4). By (13), we conclude that

$$
\begin{aligned}
& \left(R_{q^{-n}} f_{\xi}\right)(x, z) \\
= & \int_{-\infty}^{\infty} \frac{\varphi(x, \lambda)}{\lambda-z} F_{\xi}(\lambda) d \varrho_{q^{-n}}(\lambda)=\int_{-\infty}^{-a} \frac{\varphi(x, \lambda)}{\lambda-z} F_{\xi}(\lambda) d \varrho_{q^{-n}}(\lambda)
\end{aligned}
$$




$$
\begin{aligned}
& +\int_{-a}^{a} \frac{\varphi(x, \lambda)}{\lambda-z} F_{\xi}(\lambda) d \varrho_{q^{-n}}(\lambda)+\int_{a}^{\infty} \frac{\varphi(x, \lambda)}{\lambda-z} F_{\xi}(\lambda) d \varrho_{q^{-n}}(\lambda) \\
= & I_{1}+I_{2}+I_{3},
\end{aligned}
$$

where

$$
F_{\xi}(\lambda)=\int_{0}^{q^{-\xi}} f(x) \varphi(x, \lambda) d_{q} x
$$

and $a>0$.

It follows from $\sqrt{13}$ that

$$
\begin{aligned}
\left|I_{1}\right|= & \left|\int_{-\infty}^{-a} \frac{\varphi(x, \lambda)}{\lambda-z} F_{\xi}(\lambda) d \varrho_{q^{-n}}(\lambda)\right| \\
\leq & \sum_{\lambda_{k, q^{-n}}<-a} \frac{\left|\varphi_{k, q^{-n}}(x)\right|\left|\int_{0}^{q^{-\xi}} f_{\xi}(t) \varphi_{k, q^{-n}}(t) d_{q} t\right|}{\alpha_{k, q^{-n}}^{2}\left|\lambda_{k, q^{-n}}-z\right|} \\
\leq & \left(\sum_{\lambda_{k, q^{-n}<-a}} \frac{\varphi_{k, q^{-n}}^{2}(x)}{\alpha_{k, q^{-n}}^{2}\left|\lambda_{k, q^{-n}}-z\right|^{2}}\right)^{1 / 2} \\
& \times\left(\sum_{\lambda_{k, q^{-n}<-a}} \frac{1}{\alpha_{k, q^{-n}}^{2}}\left[\int_{0}^{q^{-\xi}} f_{\xi}(x) \varphi_{k, q^{-n}}(x) d_{q} x\right]^{2}\right)^{1 / 2} .
\end{aligned}
$$

Using the $q$-integration-by-parts formula in the integral below, we have

$$
\begin{aligned}
& \int_{0}^{q^{-\xi}} f_{\xi}(x) \varphi_{k, q^{-n}}(x) d_{q} x \\
= & \frac{1}{\lambda_{k, q^{-n}}} \int_{0}^{q^{-\xi}} f_{\xi}(x)\left\{-\frac{1}{q} D_{q^{-1}} D_{q} \varphi_{k, q^{-n}}(x)+r(x) \varphi_{k, q^{-n}}(x)\right\} d_{q} x \\
= & \frac{1}{\lambda_{k, q^{-n}}} \int_{0}^{q^{-\xi}}\left\{-\frac{1}{q} D_{q^{-1}} D_{q} f_{\xi}(x)+r(x) f_{\xi}(x)\right\} \varphi_{k, q^{-n}}(x) d_{q} x .
\end{aligned}
$$

From Lemma 3, we get

$$
\left|I_{1}\right| \leq \frac{K^{1 / 2}}{a}\left(\times\left[\int_{0}^{q^{-\xi}}\left\{-\frac{1}{q} D_{q^{-1}} D_{q} f_{\xi}(x)+r(x) f_{\xi}(x)\right\} \varphi_{k, q^{-n}}(x) d_{q} x\right]^{2}\right)^{1 / 2} .
$$


Application of Bessel inequality yields

$$
\left|I_{1}\right| \leq \frac{K^{1 / 2}}{a}\left[\int_{0}^{q^{-\xi}}\left\{-\frac{1}{q} D_{q^{-1}} D_{q} f_{\xi}(x)+r(x) f_{\xi}(x)\right\}^{2} d_{q} x\right]^{1 / 2}=\frac{C}{a} .
$$

Likewise, we show that $\left|I_{3}\right| \leq \frac{C}{a}$. Then $I_{1}, I_{3} \rightarrow 0$, as $a \rightarrow \infty$, uniformly in $q^{-n}$. By virtue of (19) and Theorem 4, we see that

$$
\left(R f_{\xi}\right)(x, z)=\int_{-\infty}^{\infty} \frac{\varphi(x, \lambda)}{\lambda-z} F_{\xi}(\lambda) d \varrho(\lambda) .
$$

We can find a sequence $\left\{f_{\xi}(x)\right\}_{\xi=1}^{\infty}$ which satisfies the previous conditions and tend to $f(x)$ as $\xi \rightarrow \infty$, since $f(.) \in L_{q}^{2}[0, \infty)$. It follows from 9 that the sequence of Fourier transform converges to the transform of $f(x)$. Using Lemmas 5 and 7, one can pass to the limit $\xi \rightarrow \infty$ in 22 .

Remark 9. The following formula holds.

$$
\int_{0}^{\infty}(R f)(x, z) g(x) d_{q} x=\int_{-\infty}^{\infty} \frac{F(\lambda) G(\lambda)}{\lambda-z} d \varrho(\lambda),
$$

where

and

$$
G(\lambda)=\lim _{\xi \rightarrow \infty} \int_{0}^{q^{-\xi}} g(x) \varphi(x, \lambda) d_{q} x
$$

$$
F(\lambda)=\lim _{\xi \rightarrow \infty} \int_{0}^{q^{-\xi}} f(x) \varphi(x, \lambda) d_{q} x .
$$

Now, we will study some properties of the resolvent operator. We give the following definition and theorems.

Definition 10. Let $M(x, t)$ be a complex-valued function, where $x, t \in(a, b)$. If

$$
\int_{a}^{b} \int_{a}^{b}|M(x, t)|^{2} d_{q} x d_{q} t<+\infty,
$$

then $M(x, t)$ is called the q-Hilbert-Schmidt kernel.

Theorem 11 ( 22$])$. Let us define the operator $A$ as

$$
A\left\{x_{i}\right\}=\left\{y_{i}\right\},
$$

where

$$
y_{i}=\sum_{k=1}^{\infty} a_{i k} x_{k}, i \in \mathbb{N} .
$$

If

$$
\sum_{i, k=1}^{\infty}\left|a_{i k}\right|^{2}<+\infty
$$

then $A$ is a compact operator in the sequence space $l^{2}$. 
Theorem 12. Let the limit circle case holds for Eq. (3) and

$$
G(x, t)=G(x, t, 0)= \begin{cases}\varphi(x) \chi(t), & x<t \\ \chi(x) \varphi(t), & x \geq t .\end{cases}
$$

Then the function $G(x, t)$ defined by (26) is a q-Hilbert-Schmidt kernel.

Proof. It follows from (26) that

$$
\int_{0}^{\infty} d_{q} x \int_{0}^{x}|G(x, t)|^{2} d_{q} t<+\infty
$$

and

since the integrals

$$
\int_{0}^{\infty} d_{q} x \int_{x}^{\infty}|G(x, t)|^{2} d_{q} t<+\infty
$$

$$
\int_{0}^{\infty}|G(x, t)|^{2} d_{q} x
$$

and

$$
\int_{0}^{\infty}|G(x, t)|^{2} d_{q} t
$$

exist and are a linear combination of the products $\varphi(x) \chi(t)$, and these products belong to $L_{q}^{2}[0, \infty) \times L_{q}^{2}[0, \infty)$. Then

$$
\int_{0}^{\infty} \int_{0}^{\infty}|G(x, t)|^{2} d_{q} x d_{q} t<+\infty .
$$

Theorem 13. Let us define the operator $R$ as

$$
(R f)(x)=\int_{0}^{\infty} G(x, t) f(t) d_{q} t
$$

Under the assumptions of Theorem 12, $R$ is a compact operator.

Proof. Let $\phi_{i}=\phi_{i}(t)(i \in \mathbb{N})$ be a complete, orthonormal basis of $L_{q}^{2}[0, \infty)$. By Theorem 12, we can define

$$
\begin{aligned}
x_{i} & =\left(f, \phi_{i}\right)=\int_{0}^{\infty} \overline{\phi_{i}(t)} f(t) d_{q} t, \\
y_{i} & =\left(g, \phi_{i}\right)=\int_{0}^{\infty} \overline{\phi_{i}(t)} g(t) d_{q} t, \\
a_{i k} & =\int_{0}^{\infty} \int_{0}^{\infty} \overline{\phi_{k}(t) \phi_{i}(x)} G(x, t) d_{q} x d_{q} t,
\end{aligned}
$$

where $i, k \in \mathbb{N}$. Then, $L_{q}^{2}[0, \infty)$ is mapped isometrically $l^{2}$. Therefore, the operator $R$ transforms into $A$ defined by (24) in $l^{2}$ by this mapping, and 27$)$ is translated into 25). It follows from Theorem 11 that $A$ is compact operator. Consequently, $R$ is a compact operator. 
Now, we will give some auxiliary lemmas.

Lemma 14. The following equalities hold.

$$
\begin{aligned}
\lim _{x \rightarrow \infty} W_{q}\left(\chi(x, \lambda), \chi\left(x, \lambda^{\prime}\right)\right) & =0, \\
\int_{0}^{\infty} \chi(x, \lambda), \chi\left(x, \lambda^{\prime}\right) d_{q} x & =\frac{m(\lambda)-m\left(\lambda^{\prime}\right)}{\lambda-\lambda^{\prime}},
\end{aligned}
$$

where $\lambda$ and $\lambda^{\prime}$ are any fixed nonreal numbers.

Proof. See 9 .

Using 29 and setting $\lambda=u+i v$ and $\lambda^{\prime}=\bar{\lambda}$, we obtain

$$
\int_{0}^{\infty}|\chi(x, \lambda)|^{2} d_{q} x=-\frac{\operatorname{Im}\{m(\lambda)\}}{v} .
$$

Lemma 15. For fixed $u_{1}$ and $u_{2}$, we have

$$
\int_{u_{1}}^{u_{2}}-\operatorname{Im}\{m(u+i \delta)\} d u=O(1), \text { as } \delta \rightarrow 0 .
$$

Proof. Let $\sin \beta \neq 0$. It follows from (9) and $(18)$ that

$$
\int_{0}^{\infty}|\chi(t, z)|^{2} d_{q} t=\int_{-\infty}^{\infty} \frac{d \varrho(\lambda)}{(u-\lambda)^{2}+v^{2}}
$$

where $z=u+i v$.

Let $\sin \beta=0$. If the equality $(15)$ is $q$-differentiated throughout with respect to $x$, and the limit is taken as $n \rightarrow \infty$, then we can get the desired result.

By virtue of (30) and (32), we conclude that

$$
-\operatorname{Im}\{m(u+i \delta)\}=\delta \int_{-\infty}^{\infty} \frac{d \varrho(\lambda)}{(u-\lambda)^{2}+\delta^{2}} .
$$

Then we have

$$
-\int_{u_{1}}^{u_{2}} \operatorname{Im}\{m(u+i \delta)\} d u=\delta \int_{u_{1}}^{u_{2}} d u \int_{-\infty}^{\infty} \frac{d \varrho(\lambda)}{(u-\lambda)^{2}+\delta^{2}} .
$$

Let $(a, b)$ be a finite interval where $a<u_{1}$ and $b>u_{2}$. From (17), we see that

$$
\begin{gathered}
\delta \int_{u_{1}}^{u_{2}} d u \int_{-\infty}^{a} \frac{d \varrho(\lambda)}{(u-\lambda)^{2}+\delta^{2}}=O(1), \\
\delta \int_{u_{1}}^{u_{2}} d u \int_{b}^{\infty} \frac{d \varrho(\lambda)}{(u-\lambda)^{2}+\delta^{2}}=O(1) .
\end{gathered}
$$

Hence, we get

$$
\delta \int_{u_{1}}^{u_{2}} d u \int_{a}^{b} \frac{d \varrho(\lambda)}{(u-\lambda)^{2}+\delta^{2}}=\int_{a}^{b} d \varrho(\lambda) \int_{\frac{u_{1}-\lambda}{\delta}}^{\frac{u_{2}-\lambda}{\delta}} \frac{d v}{1+v^{2}}=O(1) .
$$


Assume that $\sigma(\lambda)=\sigma_{1}(\lambda)+i \sigma_{2}(\lambda)$ is a complex bounded variation on the entire line. Set

$$
\begin{aligned}
\varphi(z) & =\int_{-\infty}^{\infty} \frac{d \sigma(\lambda)}{\lambda-z}, \psi(\sigma, \tau)=\frac{\operatorname{sgn} \tau}{\pi} \frac{\varphi(z)-\varphi(\bar{z})}{2 i} \\
& =-\frac{1}{\pi} \int_{-\infty}^{\infty} \frac{|\tau| d \sigma(\lambda)}{(\lambda-\sigma)^{2}+\tau^{2}}, z=\sigma+i \tau .
\end{aligned}
$$

Theorem 16 ( $[20])$. Let the points $a, b$ are points of continuity of $\sigma(\lambda)$. Then we obtain

$$
\sigma(b)-\sigma(a)=\lim _{\tau \rightarrow 0} \int_{a}^{b}-\psi(\sigma, \tau) d \sigma .
$$

Theorem 17. Let the endpoints of $\Delta=(\lambda, \lambda+\Delta)$ be the points of continuity of $\varrho(\lambda)$. Then, we deduce that

$$
\varrho(\lambda+\Delta)-\varrho(\lambda)=\frac{1}{\pi} \lim _{\delta \rightarrow 0} \int_{\Delta}-\operatorname{Im}\{m(u+i \delta)\} d u .
$$

Proof. Let $f(),. g(.) \in L_{q}^{2}[0, \infty)$ vanish outside a finite interval. By $(23)$, we deduce that

$$
\begin{aligned}
y(\lambda) & =\int_{0}^{\infty}(R f)(x, z) g(x) d_{q} x \\
& =\int_{-\infty}^{\infty} \frac{F(\lambda) G(\lambda)}{\lambda-z} d \varrho(\lambda)=\int_{-\infty}^{\infty} \frac{d \rho(\lambda)}{\lambda-z},
\end{aligned}
$$

where

$$
\rho(\Delta)=\int_{\Delta} F(\lambda) G(\lambda) d \varrho(\lambda) .
$$

It follows from Theorem 16 that

$$
\rho(\Delta)=-\frac{1}{\pi} \lim _{\delta \rightarrow 0} \int_{\Delta} \operatorname{Im}\{\psi(u+i \delta)\} d u .
$$

Furthermore, we have

$$
\begin{aligned}
& \operatorname{Im}\{\psi(u+i \delta)\}=\int_{0}^{\infty} g(x) d_{q} x \\
& \times\left\{\int_{0}^{x}[\theta(x, u+i \delta)+m(u+i \delta) \varphi(x, u+i \delta)] \varphi(t, u+i \delta) f(t) d_{q} t\right. \\
& \left.+\int_{x}^{\infty}[\theta(t, u+i \delta)+m(u+i \delta) \varphi(t, u+i \delta)] \varphi(x, u+i \delta) f(t) d_{q} t\right\},
\end{aligned}
$$

where $\theta(x, u), \varphi(x, u), g(x)$ and $f(x)$ are real-valued functions. It follows from (34) and Lemma 15 that

$$
\rho(\Delta)=\frac{1}{\pi} \lim _{\delta \rightarrow 0} \int_{\Delta}-\operatorname{Im}\{m(u+i \delta)\} G(u) F(u) d u .
$$


If we choose $g(x)$ and $f(x)$ conveniently, we can make $G(u)$ and $F(u)$ differ as little from unity in the fixed interval $\Delta$. From Lemma 15 and $(33)$, we get the desired result.

Theorem 18. Let $z \notin \mathbb{R}$. Then we have

$$
m(z)=-\cot \beta+\int_{-\infty}^{\infty} \frac{d \varrho(\lambda)}{\lambda-z} .
$$

Proof. It follows from (18) that

$$
G(x, t, z)=\int_{-\infty}^{\infty} \frac{\varphi(x, \lambda) \varphi(t, \lambda) d \varrho(\lambda)}{\lambda-z},
$$

since $f(x)$ is an arbitrary function. By definition, we get

$$
G(x, t, z)= \begin{cases}{[\theta(t, z)+m(z) \varphi(t, z)] \varphi(x, z),} & t>x \\ {[\theta(x, z)+m(z) \varphi(x, z)] \varphi(t, z),} & t \leq x\end{cases}
$$

By virtue of (6) and (37), we conclude that

$$
\begin{aligned}
G(0,0, z) & =\sin \beta\{\cos \beta+m(z) \sin \beta\} \\
& =\int_{-\infty}^{\infty} \frac{\sin ^{2} \beta}{\lambda-z} d \varrho(\lambda),
\end{aligned}
$$

i.e.,

$$
m(z)=-\cot \beta+\int_{-\infty}^{\infty} \frac{d \varrho(\lambda)}{\lambda-z}
$$

Authors Contribution Statement All authors jointly worked on the results and they read and approved the final manuscript.

Declaration of Competing Interests The authors declare that there is no competing interest.

\section{REFERENCES}

[1] Aldwoah, K. A., Malinowska, A. B., Torres, D. F. M., The power quantum calculus and variational problems, Dyn. Contin. Discrete Impuls. Syst., Ser. B, Appl. Algorithms, 19 (2012), 93-116.

[2] Allahverdiev, B. P., Tuna, H., A representation of the resolvent operator of singular Hahn-Sturm-Liouville problem, Numer. Funct. Anal. Optimiz., 41(4) (2020), 413-431. doi:10.1080/01630563.2019.1658604

[3] Allahverdiev, B. P., Tuna, H., An expansion theorem for $q$-Sturm-Liouville operators on the whole line, Turk. J. Math., 42 (2018), 1060-1071. doi:10.3906/mat-1705-22

[4] Allahverdiev, B. P., Tuna, H., Eigenfunction expansion in the singular case for $q$-Sturm-Liouville operators, Caspian J. Math. Sci., 8(2) (2019), 91-102 doi:10.22080/CJMS.2018.13943.1339 
[5] Allahverdiev, B. P., Tuna, H., Some properties of the resolvent of Sturm-Liouville operators on unbounded time scales, Mathematica, 61 (84) No. 1 (2019), 3-21. doi:10.24193/mathcluj.2019.1.01

[6] Allahverdiev, B. P., Tuna, H., Spectral theory of singular Hahn difference equation of the Sturm-Liouville type, Commun. Math., 28(1) (2020), 13-25. doi:10.2478/cm-2020-0002

[7] Allahverdiev, B. P., Tuna, H., On the resolvent of singular Sturm-Liouville operators with transmission conditions, Math. Meth. Appl. Sci., 43 (2020), 4286- 4302. doi:10.1002/mma.6193

[8] Annaby, M. H., Mansour, Z. S., $q$-Fractional calculus and equations. Lecture Notes in Mathematics, vol. 2056, Springer, Berlin, 2012. doi:10.1007/978-3-642-30898-7

[9] Annaby, M. H., Mansour, Z. S., Soliman, I. A., $q$-Titchmarsh-Weyl theory: series expansion, Nagoya Math. J., 205 (2012), 67-118. doi:10.1215/00277630-1543787

[10] Annaby, M. H., Mansour, Z. S., Basic Sturm-Liouville problems, J. Phys. A, Math. Gen., 38(17) (2005), 3775-3797. doi:10.1088/0305-4470/38/17/005

[11] Annaby, M. H., Hamza, A. E., Aldwoah, K. A., Hahn difference operator and associated Jackson-Nörlund integrals, J. Optim. Theory Appl., 154 (2012), 133-153. doi:10.1007/s10957012-9987-7

[12] Ernst, T., The History of $q$-Calculus and a New Method, U. U. D. M. Report (2000): 16, ISSN1101-3591, Department of Mathematics, Uppsala University, 2000.

[13] Hahn, W., Beitraäge zur Theorie der Heineschen Reihen, Math. Nachr. 2 (1949), 340-379 (in German). doi:10.1002/mana.19490020604

[14] Hamza, A. E., Ahmed, S. M., Existence and uniqueness of solutions of Hahn difference equations, Adv. Differ. Equ., 316 (2013), 1-15. doi:10.1186/1687-1847-2013-316

[15] Hamza, A. E., Ahmed, S. M., Theory of linear Hahn difference equations, J. Adv. Math., $4(2)$ (2013), 441-461.

[16] Jackson, F. H., On q-definite integrals, Quart. J. Pure Appl. Math., 41 (1910), 193-203.

[17] Kac, V., Cheung, P., Quantum Calculus, Springer-Verlag, Berlin Heidelberg, 2002. doi:10.1007/978-1-4613-0071-7

[18] Karahan, D., Mamedov, Kh. R., Sampling theory associated with $q$-Sturm-Liouville operator with discontinuity conditions, Journal of Contemporary Applied Mathematics, 10(2) (2020), $1-9$.

[19] Kolmogorov, A. N., Fomin, S. V., Introductory Real Analysis, Translated by R. A. Silverman, Dover Publications, New York, 1970.

[20] Levitan, B. M., Sargsjan, I. S., Sturm-Liouville and Dirac Operators. Mathematics and its Applications (Soviet Series). Kluwer Academic Publishers Group, Dordrecht, 1991 (translated from the Russian). doi:10.1007/978-94-011-3748-5

[21] Malinowska, A. B., Torres, D. F. M., The Hahn quantum variational calculus, J. Optim. Theory Appl., 147 (2010), 419-442. doi:10.1007/s10957-010-9730-1

[22] Naimark, M. A., Linear Differential Operators, 2nd edn.,1969, Nauka, Moscow; English transl. of 1st. edn., 1, 2, New York, 1968.

[23] Swamy, P. N., Deformed Heisenberg algebra:origin of q-calculus, Physica A: Statistical Mechanics and its Applications, 328, 1-2 (2003), 145-153. doi:10.1016/S0378-4371(03)00518-1

[24] Tariboon, J., Ntouyas, S. K., Quantum calculus on finite intervals and applications to impulsive difference equations, Adv. Differ. Equ., 282 (2013), 1-19.doi:10.1186/1687-1847-2013-282

[25] Titchmarsh, E. C., Eigenfunction Expansions Associated with Second-Order Differential Equations, Part I. Second Edition, Clarendon Press, Oxford, 1962. 